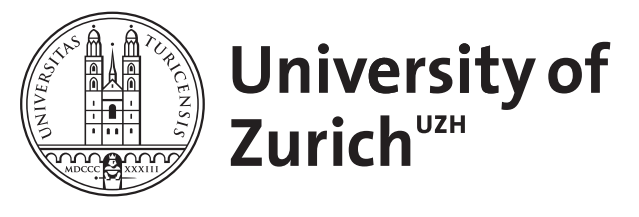

\title{
Neue Aspekte der Pathologie des Nierenzellkarzinoms
}

Moch, $\mathrm{H}$

\begin{abstract}
Die neuen Behandlungsmöglichkeiten für Nierenzellkarzinome haben das Interesse an der Histogenese und den molekularen Veränderungen von Nierentumoren erheblich verstärkt. Die aktuelle Klassifikation der Nierentumoren beruht auf spezifischen molekularen Veränderungen und charakteristischen biologischen Eigenschaften. Es wurden in den letzten Jahren mehrere neue Tumortypen identifiziert, deren Kenntnis erheblichen Einfluss auf die Auswahl der Behandlungsstrategien hat. In der vorliegenden Übersichtsarbeit wird die aktuelle Nomenklatur der Nierenneoplasien unter besonderer Berücksichtigung neuer Nierentumortypen diskutiert. Die Kenntnis von Xp11-Translokationskarzinomen und Nierentumoren mit dominanter Zystenbildung ist diagnostisch relevant. Bestimmte Genotyp-Phänotyp-Korrelationen haben zunehmenden Einfluss auf die Auswahl der Therapieverfahren und die Einschätzung der Prognose. Gleichzeitig führt eine umfangreichere pathologisch-anatomische Aufarbeitung von Nephrektomiepräparaten unter besonderer Berücksichtigung des Nierenhilus zu einer korrekteren TNM-Stadienklassifikation mit genauerer Prognosevorhersage mit sich
\end{abstract}

DOI: https://doi.org/10.1007/s00761-009-1755-4

Posted at the Zurich Open Repository and Archive, University of Zurich ZORA URL: https://doi.org/10.5167/uzh-156167

Journal Article

Published Version

Originally published at:

Moch, H (2010). Neue Aspekte der Pathologie des Nierenzellkarzinoms. Der Onkologe, 16(2):131-139.

DOI: https://doi.org/10.1007/s00761-009-1755-4 
Onkologe 2010 · 16:131-139

DOI 10.1007/s00761-009-1755-4

Online publiziert: 27. Januar 2010

(c) Springer-Verlag 2010
H. Moch

Institut für Klinische Pathologie, Departement Pathologie, Universitätsspital Zürich

\section{Neue Aspekte der Pathologie des Nierenzellkarzinoms}

\section{Die Häufigkeit der Nierentumoren steigt $v$. $a$. in den westlichen Ländern. Es gibt nur wenige gesicherte ätiolo- gische Faktoren für Nierentumoren [1]. Neben Nikotinabusus soll das Er- nährungsverhalten einen Einfluss auf die Entwicklung von Nierentumoren haben, denn insbesondere bei adi- pösen Patienten treten Nierenkar- zinome gehäuft auf. In der Schweiz wurde errechnet, dass das relative Ri- siko für die Entstehung eines Nieren- zellkarzinoms bei adipösen Patienten bei 1,8 liegt [2].}

Wegen der sehr unterschiedlichen morphologischen Bilder von Nierenkarzinomen existierten lange kontrovers diskutierte Klassifikationssysteme. Die Nomenklatur der Nierentumoren versucht einerseits, dem histologischen Wachstumsmuster Rechnung zu tragen, andererseits aber auch Aussagen zur Prognose zu liefern bzw. molekulargenetische Veränderungen in bestimmten Nierentumortypen wiederzuspiegeln. Der Begriff Hypernephrom ist obsolet, da er unter der Annahme entstand, dass sich Nierentumoren aus versprengten Nebennierenkeimen entwickeln. Frühere Klassifikation wurden aufgrund der Zytoplasmamorphologie (klarzellig vs. granularzellig) oder des Wachstumstyps (azinär, papillär, alveolär etc.) entwickelt [3]. Dies führte zu der groben Einteilung der Nierenkarzinome in klarzellige, chromophile und chromophobe Karzinome sowie Sammelrohrkarzinome und die Abgrenzung dieser malignen Tumoren von den papillären
Adenomen und dem Onkozytom. Es wird postuliert, dass die klarzelligen und chromophilen Karzinome von den proximalen Tubulusabschnitten abstammen, die chromophoben Karzinome und Onkozytome von den distalen Tubulusabschnitten und das Duct-Bellini-Karzinom von dem Sammelrohrsystem.

\section{Aktuelle Nomenklatur der Nierentumoren}

Spätere zytogenetische und molekulare Analysen haben gezeigt, dass bestimmte genetische Veränderungen mit bestimmten Nierentumortypen assoziiert sind. Im Jahr 2004 hat die WHO die Nomenklatur der Nierenkarzinome revidiert [4]. Diese Klassifikation stützte sich auf die Kenntnisse der spezifischen molekularen Veränderungen einzelner Nierentumortypen. Dies führte zu einer Veränderung der Nomenklatur der bekannten Nierentumoren (• Tab. 1). Die chromophilen Karzinome werden nun als papilläre Karzinome bezeichnet. Damit trägt man der Tatsache Rechnung, dass bei diesen $\mathrm{Tu}$ moren in mehr als $70 \%$ der Tumorareale ein papilläres Wachstumsmuster vorliegt. Die aktuelle Nomenklatur berücksichtigt erstmals Erkenntnisse der Zytogenetik und Molekularbiologie. Dabei sind die typischen molekularen Veränderungen bei den klarzelligen Karzinomen ein Verlust des kurzen Arms von Chromosom 3 bei häufiger gleichzeitiger Mutation des Von- Hippel-Lindau- (VHL-)Gens auf Chromosom 3p25-26 [5]. Bei den papillären Karzinomen kann diese Verände- rung nicht nachgewiesen werden. Dieser Typ ist durch eine Vermehrung des Chromosoms 7 und des Chromosoms 17 bei gleichzeitigem Verlust von Chromosom-Y-Stücken charakterisiert [6]. Demgegenüber sind chromophobe Karzinome [7] durch einen Verlust von zahlreichen Chromosomenstücken gekennzeichnet, u. a. der Chromosomen 1, 3, 4, 7, 9, 12, 13 und 16 [8]. Die genetischen Veränderungen von Sammelrohrkarzinomen sind bislang nur unzureichend charakterisiert, da dieser Tumortyp sehr selten ist. Da aktuelle „targeted therapies“ beispielsweise die VHL-HIF-Signalwege beim klarzelligen Nierenzellkarzinom beeinflussen (s. andere Beiträge in diesem Themenheft), haben die Nomenklatur und die exakte Zuordnung der Nierentumortypen prognostische Bedeutung. Der häufigste Tumortyp ist das klarzellige Nierenzellkarzinom [9], der damit mehrheitlich auch die aktuellen klinisch-onkologischen Studien dominiert. Eine kürzlich erstellte Zusammenfassung der Literaturangaben zu Biomarkern in Nierenkarzinomen beinhaltet demzufolge auch im Wesentlichen Angaben zu diesem Tumortyp [10].

\section{Kenntnis neuer \\ Nierentumortypen als \\ Voraussetzung der individualisierten Therapie}

Neben den o. g. häufigen Nierenkarzinomtypen wurden in den letzten Jahren weitere seltene Nierentumoren identifiziert, die nunmehr in die neue WHO-Klassifikation aufgenommen wurden. Die nächs- 


\begin{tabular}{|c|c|}
\hline \multicolumn{2}{|c|}{ Epitheliale Nierenzelltumoren } \\
\hline Maligne & Benigne \\
\hline Klarzellliges Karzinom & $\begin{array}{l}\text { Papilläres Ade- } \\
\text { nom }\end{array}$ \\
\hline Papilläres Karzinom & Onkozytom \\
\hline \multicolumn{2}{|l|}{ Chromophobes Karzinom } \\
\hline \multicolumn{2}{|l|}{ Sammelrohrkarzinom } \\
\hline \multicolumn{2}{|l|}{$\begin{array}{l}\text { Medulläres Karzinom der } \\
\text { Niere }\end{array}$} \\
\hline \multicolumn{2}{|l|}{$\begin{array}{l}\text { Karzinom assoziiert mit } \\
\text { Neuroblastom }\end{array}$} \\
\hline \multicolumn{2}{|l|}{$\begin{array}{l}\text { Muzinöses tubuläres und } \\
\text { spindelzelliges Karzinom }\end{array}$} \\
\hline \multicolumn{2}{|l|}{$\begin{array}{l}\text { TFE-3-Translokationskar- } \\
\text { zinom }\end{array}$} \\
\hline \multicolumn{2}{|l|}{ Metanephrische Tumoren } \\
\hline \multicolumn{2}{|l|}{ Metanephrisches Adenom } \\
\hline \multicolumn{2}{|c|}{ Metanephrisches Adenofibrom } \\
\hline \multicolumn{2}{|c|}{ Metanephrischer Stromatumor } \\
\hline \multicolumn{2}{|l|}{ Nephroblastische Tumoren } \\
\hline \multicolumn{2}{|l|}{ Nephrogene Reste } \\
\hline \multicolumn{2}{|l|}{ Nephroblastom } \\
\hline \multicolumn{2}{|l|}{ Mesenchymale Tumoren } \\
\hline Kindesalter & Erwachsene \\
\hline Klarzellsarkom & $\begin{array}{l}\text { Sarkome und } \\
\text { benigne mes- } \\
\text { enchymale } \\
\text { Tumoren }\end{array}$ \\
\hline Mesoblastisches Nephrom & $\begin{array}{l}\text { Angiomyoli- } \\
\text { pom }\end{array}$ \\
\hline Rhabdoidtumor & $\begin{array}{l}\text { Juxtaglomeru- } \\
\text { lärerTumor }\end{array}$ \\
\hline \multicolumn{2}{|c|}{$\begin{array}{l}\text { Gemischte mesenchymale und epitheliale } \\
\text { Tumoren }\end{array}$} \\
\hline \multicolumn{2}{|l|}{$\begin{array}{l}\text { Zystisches Nephrom/gemis } \\
\text { Stromatumor }\end{array}$} \\
\hline \multicolumn{2}{|l|}{ Synoviales Sarkom } \\
\hline \multicolumn{2}{|l|}{ Neuroendokrine Tumoren } \\
\hline \multicolumn{2}{|l|}{ Karzinoid } \\
\hline \multicolumn{2}{|l|}{ Neuroendokrines Karzinom } \\
\hline \multicolumn{2}{|c|}{ Primitiver neuroektodermaler Tumor (PNET) } \\
\hline \multicolumn{2}{|l|}{ Neuroblastom } \\
\hline \multicolumn{2}{|l|}{ Phäochromozytom } \\
\hline \multicolumn{2}{|c|}{ Keimzelltumoren und Lymphome } \\
\hline Metastasen & \\
\hline
\end{tabular}

te WHO Klassifikation wird voraussichtlich weitere Entitäten enthalten. $\mathrm{Zu}$ nennen sind das tubulozystische Nierenkarzinom [11] und Nierenkarzinome in Endstadiumnieren [12]. Die Kenntnis dieser neuen Nierentumortypen ist von praktischer Bedeutung, da diese Tumoren zwar selten sind, aber die richtige Diagnose bei sol-
Tab. 2 Translokationsassoziierte Nierentumoren

Ewing-Sarkom

(PNET)

Synoviales Sarkom $\quad t(X, 18)(p 11 ; q 11)$

Mesoblastisches $\quad t(12,15)(q 13 ; q 25)$

Nephrom $^{\text {a }}$

Klarzelliges Sarkom ${ }^{\mathrm{a}} \quad t(11,22)(q 13 ; q 12)$

Nierenkarzinome mit $t(X ; 17)(q 11.2 ; q 25)$

Translokation $^{\mathrm{a}} \quad \mathrm{t}(6,11)(\mathrm{p} 21 ; \mathrm{q} 12)$

${ }^{a}$ Vorwiegend im Kindesalter.

Tab. 3 Nierentumoren mit charakteristischer Zystenbildung

Multilokuläres zystisches Nierenkarzinom

Gemischter Epithel- und Stromatumor/zystisches Nephrom

Synoviales Sarkom (zystisches embryonales Sarkom)

Tubulozystisches Nierenzellkarzinom

Nierenkarzinome in zystischen Endstadiumnieren

chen Fällen eine wesentliche Änderung der Therapie bedeuten kann. Durch die Beschreibung dieser Tumoren werden sie in den letzten Jahren auch häufiger diagnostiziert. Zu nennen sind dabei in erster Linie die Translokationskarzinome bei Kindern und Jugendlichen (• Abb. 1), die synovialen Karzinome (• Abb. 2), die muzinösen tubulären und spindelzelligen Karzinome [13] und die primitiven neuroektodermalen Tumoren der Niere. Die Beispiele des Synovialsarkoms und des primitiven neuroektodermalen Tumors (PNET/Ewing-Sarkom) der Nieren stehen für zwei seltene Tumortypen, die normalerweise im Weichgewebe bzw. im Knochen auftreten. Beide Tumoren haben spezifische Translokationen. PNET der Niere sind charakterisiert durch die Translokation $\mathrm{t}(11 ; 22)$ (q24; q12) mit dem Fusionstranskript des EWS-Gens (22q12) und dem ETS-assoziierten Onkogen FLI 1 (11q24) [14, 15]. Synoviale Sarkome haben die Translokation $\mathrm{t}(\mathrm{X} ; 18)$ mit dem Fusionstranskript SYT-SSX, das durch eine RT-PCR-Untersuchung nachgewiesen werden kann [16]. Früher wurden wahrscheinlich einzelne Fälle von synovialen Sarkomen als adulte Wilms-Tumoren diagnostiziert. Mit der zunehmenden Verfügbarkeit der molekularen Techniken werden in den letzten Jahren vermehrt synoviale Sarkome in verschiedenen Lo- kalisationen, u. a. in der Niere, nachgewiesen. Eine kürzliche Zusammenstellung von synovialen Sarkomen der Niere zeigt, dass die Patienten häufig zwischen 20 und 40 Jahre alt sind [17]. Rezidive sind nicht selten. Bei entsprechendem Einsatz einer spezifischen Chemotherapie sind teilweise günstige Verläufe zu beobachten. Synoviale Sarkome und PNET/Ewing-Sarkome der Niere haben somit charakteristische Translokationen und erweitern das Spektrum der Nierentumoren mit Translokationen. Eine Übersicht über solche translokationsassoziierten Nierentumoren wird in - Tab. 2 gegeben. Erwähnenswert in dieser Gruppe der translokationsassoziierten Tumoren ist der sog. Xp11.2-Translokationstumor $[18,19,20]$. Dieser Tumortyp wurde erst vor kurzem beschrieben und tritt im Wesentlichen im Kindes- bzw. jungen Erwachsenenalter auf. Die meisten Patienten haben ein Alter zwischen 4 und 19 Jahren [21]. Eine von uns durchgeführte Analyse von Nierentumoren im Kindes- und jungen Erwachsenenalter unter Einbezug von Fällen aus dem KinderTumor-Referenzzentrum in Kiel hat gezeigt, dass dieser Tumortyp in diesem Alter vergleichsweise häufig ist. Ungewöhnlich für diesen Tumortyp ist ein papilläres Wachstum, wobei die Zellen ein klarzelliges Zytoplasma aufweisen. Diese Tumoren haben unterschiedliche Translokationen, die jedoch alle in eine Genfusion des Gens TFE-3 münden. Häufige Translokationen sind $\mathrm{t}(\mathrm{X} ; 1)(\mathrm{p} 11.2 ; \mathrm{q} 21)$ sowie die Translokation $t(X ; 17)(p 11.2 ; q 25)$. Diese resultieren in eine Fusion des ASPL- und TFE-3-Gens [22]. Interessanterweise werden solche Translokationen auch bei sog. alveolären Weichgewebssarkomen beobachtet. Die Morphologie dieser Nierentumoren ist typisch. Über die Prognose der Patienten mit Translokationskarzinomen ist bislang nur wenig bekannt. Eine jüngere Arbeit hat gezeigt, dass diese Tumoren auch im Erwachsenenalter auftreten können und die Prognose ungünstig ist [23]. Zahlreiche dieser Karzinome präsentieren sich schon mit lymphogenen oder hämatogenen Metastasen. 
Hier steht eine Anzeige.

算 Springer 
Onkologe 2010 • 16:131-139 DOI 10.1007/s00761-009-1755-4

(c) Springer-Verlag 2010

\title{
H. Moch \\ Neue Aspekte der Pathologie des Nierenzellkarzinoms
}

\begin{abstract}
Zusammenfassung
Die neuen Behandlungsmöglichkeiten für Nierenzellkarzinome haben das Interesse an der Histogenese und den molekularen Veränderungen von Nierentumoren erheblich verstärkt. Die aktuelle Klassifikation der Nierentumoren beruht auf spezifischen molekularen Veränderungen und charakteristischen biologischen Eigenschaften. Es wurden in den letzten Jahren mehrere neue Tumortypen identifiziert, deren Kenntnis erheblichen Einfluss auf die Auswahl der Behandlungsstrategien hat. In der vorliegenden Übersichtsarbeit wird die aktuelle Nomenklatur der Nierenneoplasien unter besonderer Berücksichtigung neuer Nierentumortypen diskutiert. Die Kenntnis von Xp11-Transloka-
\end{abstract}

tionskarzinomen und Nierentumoren mit dominanter Zystenbildung ist diagnostisch relevant. Bestimmte Genotyp-Phänotyp-Korrelationen haben zunehmenden Einfluss auf die Auswahl der Therapieverfahren und die Einschätzung der Prognose. Gleichzeitig führt eine umfangreichere pathologisch-anatomische Aufarbeitung von Nephrektomiepräparaten unter besonderer Berücksichtigung des Nierenhilus zu einer korrekteren TNMStadienklassifikation mit genauerer Prognosevorhersage mit sich.

Schlüsselwörter Klassifikation · Tumorstadium · Histologie · Prognose $\cdot$ Nierenzysten

\section{New aspects of the pathology of renal cell cancer}

\begin{abstract}
New treatment options for renal cell cancer have increased the interest in the histogenesis and in molecular alterations of renal tumors. The current WHO classification of renal cancer is based on specific molecular alterations and characteristic biologic behavior. During recent years novel tumor types have been identified with immediate impact for the selection of new treatment modalities. In this review the current classification of renal cancer with special emphasis on novel renal tumor types will be discussed. Knowl-
\end{abstract}

edge of Xp11 translocation carcinomas and renal tumors with characteristic cyst formation is diagnostically relevant. In addition this review focuses on reassessing the current TNM staging system for renal cell cancer. Retrospective studies have shown the relevance of renal sinus invasion for stage evaluation.

\section{Keywords}

Classification · Tumor stage · Histology · Prognosis - Renal cysts

\section{Zystische Neoplasien der Niere: Pathologie und molekulare Veränderungen}

In - Tab. 3 sind Nierentumoren mit überwiegend zystischem Wachstumsmuster zusammengestellt. Zystische Tumorentitäten, die voraussichtlich Eingang in die nächste WHO Klassifikation finden, sind das tubulozystische Nierenkarzinom und spezifische, teils zystische Nierenkarzinome in Endstadiumnieren.

Charakteristisch für synoviale Sarkome ist das zystische Wachstum. Primär wurden synoviale Sarkome der Nieren als zystische embryonale Sarkome bezeichnet [16]. Gemischte Epithelstromatumoren sind ungewöhnliche Tumoren, die vornehmlich oder fast ausschließlich bei perimenopausalen Frauen beobachtet werden [24]. Es wird postuliert, dass eine Östrogenexposition bei der Entstehung eine Rolle spielt und sie evtl. als Spielart der zystischen Nephrome anzusehen sind. Viele Fälle wurden früher wahrscheinlich unter dem Namen zystisches Hamartom oder adultes mesoblastisches Nephrom publiziert [25]. Die Prognose der gemischten Epithelstromatumoren ist offenbar sehr gut. Synovialsarkome und gemischte Epithelstromatumoren zeigen, dass in der Gruppe der zystischen Nierentumoren neben weitgehend gutartigen Tumoren auch hoch maligne Tumoren vorhanden sind.

Das multilokuläre zystische Nierenzellkarzinom gilt als Untergruppe der klarzelligen Karzinome, hat jedoch eine ausgezeichnete Prognose. Die Tatsache, dass in Nieren von Patienten mit Von-HippelLindau-Syndrom klarzellige Nierenkarzinome und Zysten nebeneinander vorkommen, und das unterschiedliche Ausmaß von Zysten innerhalb von Nierenkarzinomen bei sporadischen Karzinomen hat verschiedene Untersuchungen ausgelöst, die die Bedeutung des VHLProteins als Bestandteil der Primärzilien der Nierentubulusepithelzellen zeigten. Die Inaktivierung des VHL-Gens führt neben der Aktivierung von HIF und CX$\mathrm{CR}_{4}[26,27]$ zu einem Verlust dieser Zilien, was die Ausbildung von Zysten begünstigt $[28,29]$. Die Reduktion der Zilien wiederum aktiviert Signalwege, die die Proliferation der Nierentubuluszellen 
Hier steht eine Anzeige.

算 Springer 


\section{Leitthema}

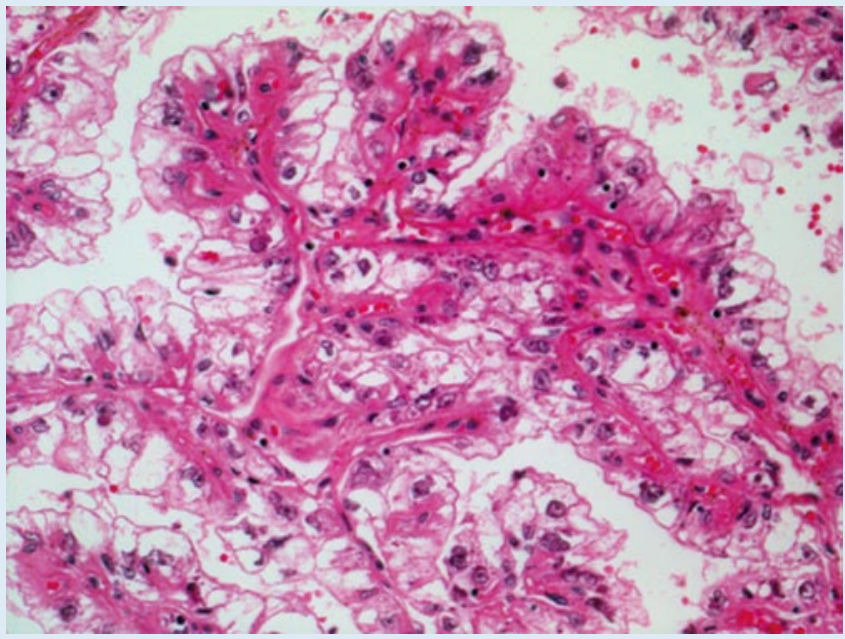

Abb. $1 \Delta$ Translokationskarzinom der Niere. Dieser Tumor tritt vorwiegend im Kindesalter auf (HE-Färbung)

Abb. 2 Synoviales Sarkom der Niere. Das zystische Wachstum (oben) ist typisch für diese Tumoren (HE-Färbung)
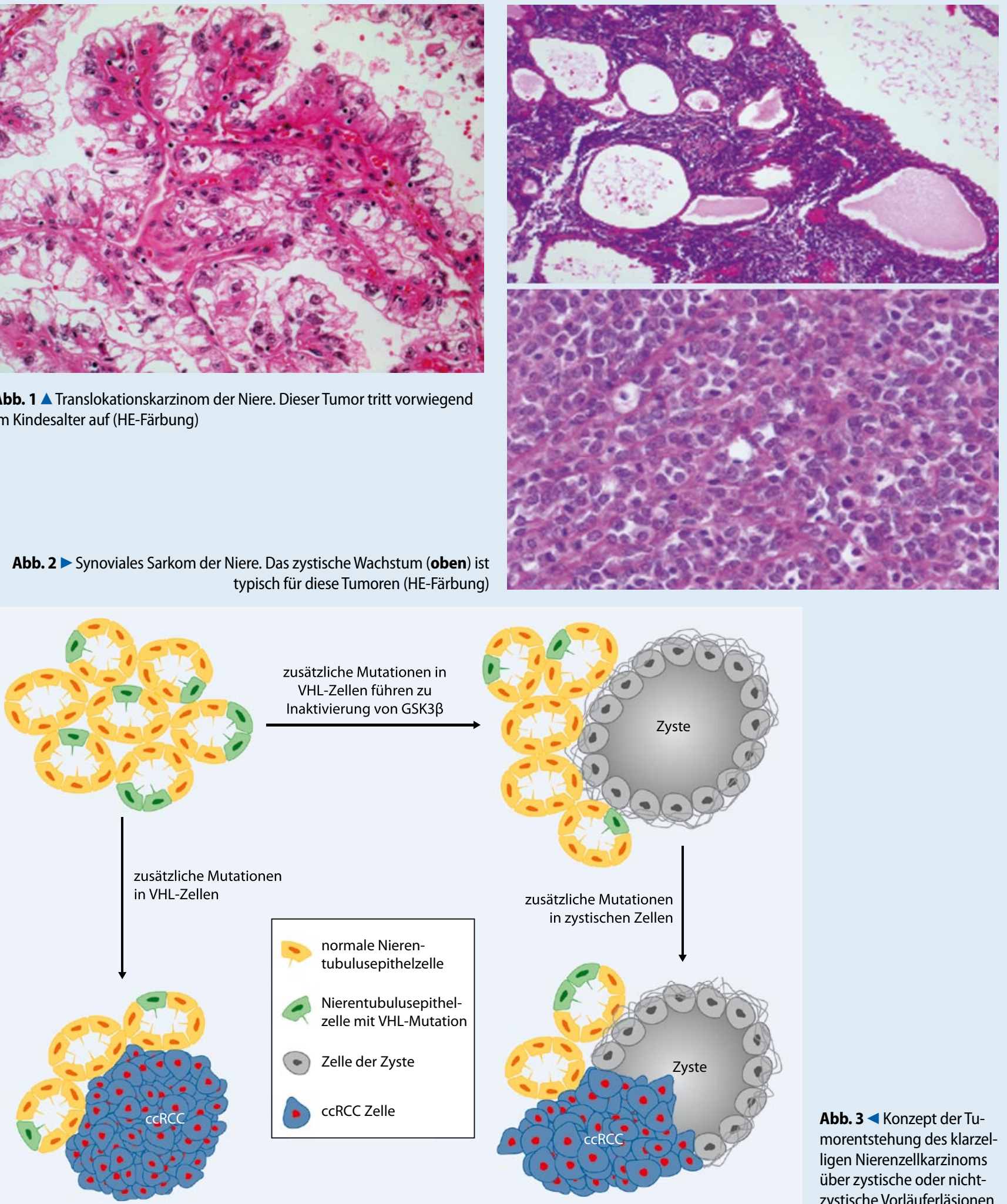

zystenunabhängiger Progressionsweg klarzelliger Nierenkarzinomzellen

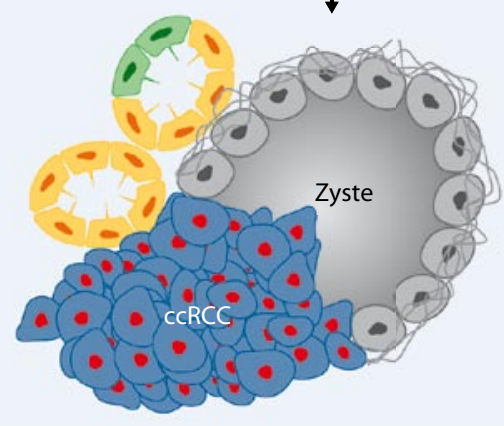

zystenabhängiger Progressionsweg klarzelliger Nierenkarzinomzellen
Abb. $3<$ Konzept der Tumorentstehung des klarzelligen Nierenzellkarzinoms über zystische oder nichtzystische Vorläuferläsionen (adaptiert nach [30]). cCRCC clear cell renal cell carcinoma 
Hier steht eine Anzeige.

算 Springer 
beschleunigen. Dieser Vorgang ist möglicherweise einer der ersten Schritte in der Tumorentstehung bei Patienten mit VHL-Syndrom. Es wird derzeit ein neues Tumorprogressionsmodell postuliert, bei dem ein zystenabhängiger und zystenunabhängiger Progressionsweg existiert ([3o], - Abb. 3).

\section{Nierenhilus: ausschlaggebend für das korrekte Tumorstadium?}

Das Tumorstadium ist neben dem histologischen Differenzierungsgrad und dem Vorliegen einer sarkomatoiden Differenzierung einer der wichtigsten prognostischen Parameter $[9,31]$. Die pT-Kategorie der Nierenkarzinome unterlag in den letzten Jahren verschiedenen Veränderungen. Vor allem die Unterscheidung zwischen $\mathrm{pT}_{1}$ - und $\mathrm{pT}_{2}$-Tumoren (basierend auf dem Tumordurchmesser) hat sich verschoben, da die verbesserte Bildgebung Nierentumoren in zunehmend früheren Stadien erfassen konnte. Die Veränderungen des Nierentumorstadiums und seine Bedeutung für die Prognose haben wir in einer kürzlichen Übersichtsarbeit, basierend auf 62 Publikationen, zusammengefasst [32]. In den vergangenen Jahren wurden wichtige Untersuchungen publiziert, die die Bedeutung der makroskopischen Aufarbeitung und histologischen Untersuchung der $\mathrm{Ne}$ phrektomiepräparate unterstreichen. Die Arbeiten von Bonsib et al. haben gezeigt [33, 34, 35], dass bei der Untersuchung des Gewebes im Nierenhilus sehr häufig Tumorzelleinbrüche in die dort befindlichen dünnwandigen Blutgefäße nachweisbar sind. Dies führt in einem hohen Prozentsatz der Fälle zu einem höheren Tumorstadium. Bei der Analyse von archivierten Fällen konnte so gezeigt werden, dass bei einem sehr großer Prozentsatz der ehemals als pT2 klassifizierten Fälle eigentlich ein $\mathrm{pT}_{3}$-Stadium mit mikroskopisch nachweisbarem Einbruch in die Hilusgefäße vorlag. Eine nachträgliche Untersuchung der Prognose zeigte, dass dies die Fälle mit ungünstigerem Verlauf waren. In der Pathologie wird daher der Aufarbeitung des Nierenhilus zunehmend Aufmerksamkeit geschenkt.

\section{Fazit für die Praxis}

Vornehmlich bei jüngeren Patienten muss an einen der seltenen Typen von Nierentumoren gedacht werden. Eine korrekte Diagnose und der spezifische Einsatz einer entsprechenden Therapie auf der Grundlage der spezifischen molekularen Veränderungen kann die Prognose bei solchen Fällen deutlich verbessern. Bei zystischen Nierentumoren ist zu beachten, dass neben Tumoren mit sehr guter Prognose (gemischter Epithelstromatumor, multilokuläres zystisches Karzinom) auch hoch maligne Tumoren in diese Kategorie fallen können (synoviales Sarkom). Der Tumorzelleinbruch in dünnwandige Blutgefäße im Bereich des Nierenhilus ist nicht selten und hat ein höheres Tumorstadium mit entsprechend ungünstigerer Prognose zur Folge.

\section{Korrespondenzadresse}

\section{Prof. Dr. H. Moch}

Institut für Klinische Pathologie, Departement Pathologie, Universitätsspital Zürich Schmelzbergstraße 12, 8091 Zürich Schweiz

Interessenkonflikt. Der korrespondierende Autor gibt an, dass kein Interessenkonflikt besteht.

\section{Literatur}

1. McLaughlin JK, Lipworth L (2000) Epidemiologic aspects of renal cell cancer. Semin Oncol 27(2):115-123

2. Ceschi M, Gutzwiller F, Moch H et al (2007) Epidemiology and pathophysiology of obesity as cause of cancer. Swiss Med Wkly 137(3-4):50-56

3. Thoenes W, Stoerkel S, Rumpelt H (1986) Histopathology and classification of renal cell tumors (adenomas, oncocytomas, and carcinomas): the basic cytological and histopathological elements and their use for diagnostics. Pathol Res Pract 181:125-143

4. Eble J, Sauter G, Epstein J, Sesterhenn I (2004) Tumours of the Kidney. In: Tumours of the Urinary System and Male Genital Organs. IARC, Lyon

5. Gnarra JR, Tory K, Weng Y et al (1994) Mutations of the VHL tumour suppressor gene in renal carcinoma. Nat Genet 7(1):85-90

6. Kovacs G, Fuzesi L, Emanual A, Kung HF (1991) Cytogenetics of papillary renal cell tumors. Genes Chromosom Cancer 3(4):249-255

7. Amin MB, Paner GP, Alvarado-Cabrero l et al (2008) Chromophobe renal cell carcinoma: histomorphologic characteristics and evaluation of conventional pathologic prognostic parameters in 145 cases. Am J Surg Pathol 32(12):1822-1834
8. Speicher M, Schoell B, Du Manoir S et al (1994) Specific loss of chromosomes 1, 2, 6, 10, 13, 17, and 21 in chromophobe renal cell carcinomas revealed by comparative genomic hybridization. Am J Pathol 145(2):356-364

9. Moch H, Gasser T, Amin MB et al (2000) Prognostic utility of the recently recommended histologic classification and revised TNM staging system of renal cell carcinoma: a Swiss experience with 588 tumors. Cancer 89(3):604-614

10. Eichelberg C, Junker K, Ljungberg B, Moch H (2009) Diagnostic and prognostic molecular markers for renal cell carcinoma: a critical appraisal of the current state of research and clinical applicability. Eur Urol 55(4):851-863

11. Amin MB, MacLennan GT, Gupta R et al (2009) Tubulocystic carcinoma of the kidney: clinicopathologic analysis of 31 cases of a distinctive rare subtype of renal cell carcinoma. Am J Surg Pathol 33(3):384-392

12. Tickoo SK, Deperalta-Venturina MN, Harik LR et al (2006) Spectrum of epithelial neoplasms in endstage renal disease: An experience from 66 tumorbearing kidneys with emphasis on histologic patterns distinct from those in sporadic adult renal neoplasia. Am J Surg Pathol 30(2):141-153

13. Rakozy C, Schmahl GE, Bogner S, Storkel S (2002) Low-grade tubular-mucinous renal neoplasms: morphologic, immunohistochemical, and genetic features. Mod Pathol 15(11):1162-1171

14. Parham DM, Roloson GJ, Feely M et al (2001) Primary malignant neuroepithelial tumors of the kidney: a clinicopathologic analysis of 146 adult and pediatric cases from the National Wilms' Tumor Study Group Pathology Center. Am J Surg Pathol 25(2):133-146

15. Casella R, Moch H, Rochlitz C et al (2001) Metastatic primitive neuroectodermal tumor of the kidney in adults. Eur Urol 39:613-617

16. Argani P, Faria PA, Epstein Jl et al (2000) Primary renal synovial sarcoma: molecular and morphologic delineation of an entity previously included among embryonal sarcomas of the kidney. Am J Surg Pathol 24(8):1087-1096

17. Moch H, Wodzynski A, Guillou L, Nickeleit V (2003) Primary renal synovial sarcoma. A new entity in the morphological spectrum of spindle cell renal tumors. Pathologe 24(6):466-472

18. Argani P, Antonescu CR, Illei PB et al (2001) Primary renal neoplasms with the ASPL-TFE3 gene fusion of alveolar soft part sarcoma: a distinctive tumor entity previously included among renal cell carcinomas of children and adolescents. Am J Pathol 159(1):179-192

19. Argani P, Antonescu CR, Couturier J et al (2002) PRCC-TFE3 renal carcinomas: morphologic, immunohistochemical, ultrastructural, and molecular analysis of an entity associated with the $t(X ; 1)(p 11.2 ; q 21)$. Am J Surg Pathol 26(12):15531566

20. Argani P, Lae M, Hutchinson B et al (2005) Renal Carcinomas with the $t(6 ; 11)(p 21 ; q 12)$ : Clinicopathologic features and demonstration of the specific alpha-TFEB gene fusion by immunohistochemistry, RT-PCR, and DNA PCR. Am J Surg Pathol 29(2):230-240

21. Bruder E, Passera O, Harms D et al (2004) Morphologic and molecular characterization of renal cell carcinoma in children and young adults. Am J Surg Pathol 28(9):1117-1132 
22. Argani P, Lal $P$, Hutchinson B et al (2003) Aberrant nuclear immunoreactivity for TFE3 in neoplasms with TFE3 gene fusions: a sensitive and specific immunohistochemical assay. Am J Surg Pathol 27(6):750-761

23. Argani P, Olgac S, Tickoo SK et al (2007) Xp11 Translocation renal cell carcinoma in adults: Expanded clinical, pathologic, and genetic spectrum. Am J Surg Pathol 31(8):1149-1160

24. Adsay NV, Eble JN, Srigley JR et al (2000) Mixed epithelial and stromal tumor of the kidney. Am J Surg Pathol 24(7):958-970

25. Moch H, Schurch LV, Sulser T, Terracciano L (2004) Mixed epithelial and stromal tumor of the kidney. Pathologe 25(5):356-361

26. Staller P, Sulitkova J, Lisztwan J et al (2003) Chemokine receptor $\mathrm{CXCR4}$ downregulated by von Hippel-Lindau tumour suppressor pVHL. Nature 425(6955):307-311

27. Frew IJ, Thoma CR, Georgiev S et al (2008) pV$\mathrm{HL}$ and PTEN tumour suppressor proteins cooperatively suppress kidney cyst formation. Embo J 27(12):1747-1757

28. Thoma CR, Frew IJ, Hoerner CR et al (2007) pVHL and GSK3beta are components of a primary cilium-maintenance signalling network. Nat Cell Biol 9(5):588-595

29. Schraml P, Frew IJ, Thoma CR et al (2009) Sporadic clear cell renal cell carcinoma but not the papillary type is characterized by severely reduced frequency of primary cilia. Mod Pathol 22(1):31-36

30. Thoma CR, Frew IJ, Krek W (2007) The VHL tumor suppressor: riding tandem with GSK3beta in primary cilium maintenance. Cell Cycle 6(15):18091813

31. Peralta-Venturina $\mathrm{M}$ de, Moch $\mathrm{H}$, Amin $\mathrm{M}$ et al (2001) Sarcomatoid differentiation in renal cell carcinoma: a study of 101 cases. Am J Surg Pathol 25(3):275-284

32. Moch H, Artibani W, Delahunt B et al (2009) Reassessing the current UICC/AJCC TNM staging for renal cell carcinoma. Eur Urol 56:636-643

33. Bonsib SM (2005) T2 clear cell renal cell carcinoma is a rare entity: a study of 120 clear cell renal cell carcinomas. J Urol 174(4 Pt 1):1199-1202; discussion 202

34. Bonsib SM (2006) Renal lymphatics, and lymphatic involvement in sinus vein invasive (pT3b) clear cell renal cell carcinoma: a study of 40 cases. Mod Pathol 19(5):746-753

35. Bonsib SM (2007) Renal veins and venous extension in clear cell renal cell carcinoma. Mod Pathol 20(1):44-53
„Der Onkologe“ bietet jeden Monat umfassende und aktuelle Beiträge zu interessanten Themenschwerpunkten aus allen Bereichen der Onkologie.

Möchten Sie ein bereits erschienenes Heft nachbestellen? Die folgenden Ausgaben können Sie direkt bei unserem Kundenservice zum Preis von je EUR 33,- beziehen:

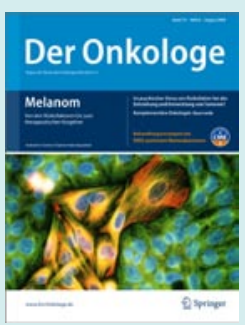

2009

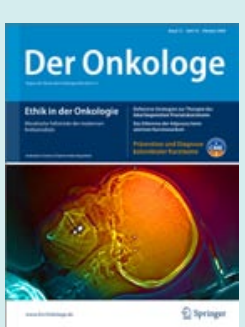

- Heft 1/09

- Heft 2/09

- Heft 3/09

- Heft 4/09

- Heft 5/09

Tumoren von Vagina und Vulva

Supportivtherapie

Seltene Tumoren

Weichteilsarkome

Vergleichende Wertigkeit bildgebender

Verfahren

- Heft 6/09

Transplantation in der Onkologie

- Heft 7/09

Tumorschmerz

- Heft 8/09

Melanom

- Heft 9/09

- Heft 10/09

Endometriumkarzinom

Ethik in der Onkologie

- Heft 11/09

Interdisziplinarität in der Onkologie

- Heft 12/09

Kolonkarzinom

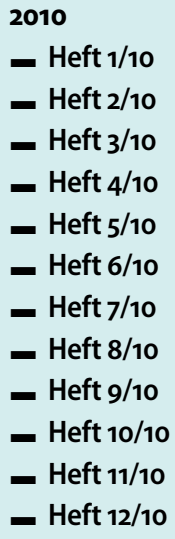

Morbus Hodgkin

Nierenzellkarzinom

Myelom

Onkologische Notfälle

Ophthalmologische Onkologie

Ösophaguskarzinom

Pankreaskarzinom

Rektumkarzinom

Schilddrüsenkarzinom

Melanom

Gallengangskarzinom

Gesundheitsökonomie

Änderungen vorbehalten
So erreichen Sie unseren Kundenservice:

Springer-Verlag

Kundenservice Zeitschriften

Haberstr. 7

69126 Heidelberg

Tel.: +49 6221 345-4303

Fax: +49 $6221345-4229$

E-Mail: leserservice@springer.com

www.DerOnkologe.de 